EDITORA

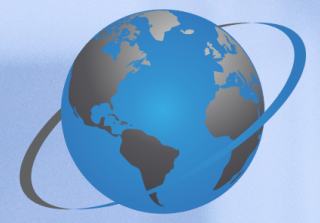

OMNIS SCIENTIA

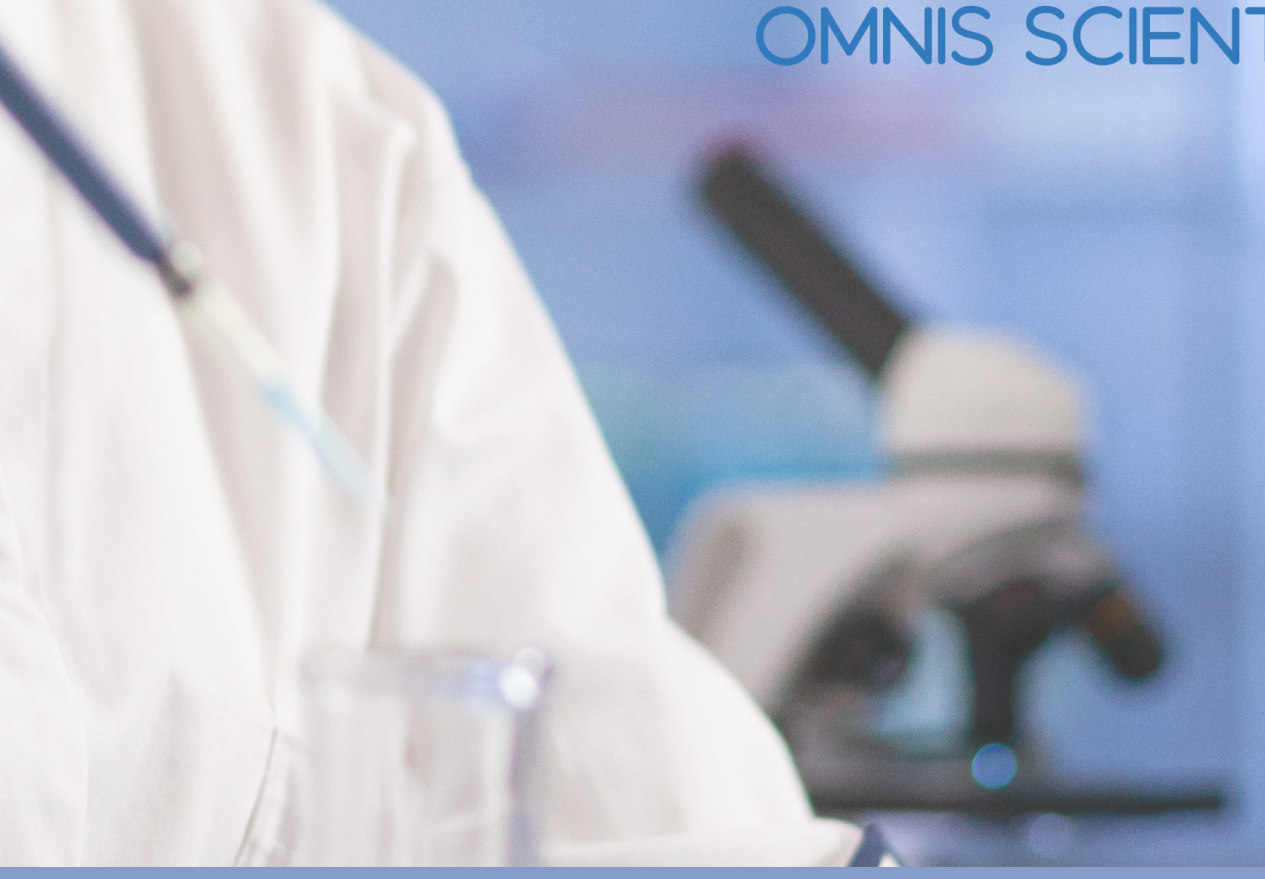

SAÚDE PÚBLIGA

NO SÉCULO XXI:

\title{
UMA ABORDAGEM SOBRE
}

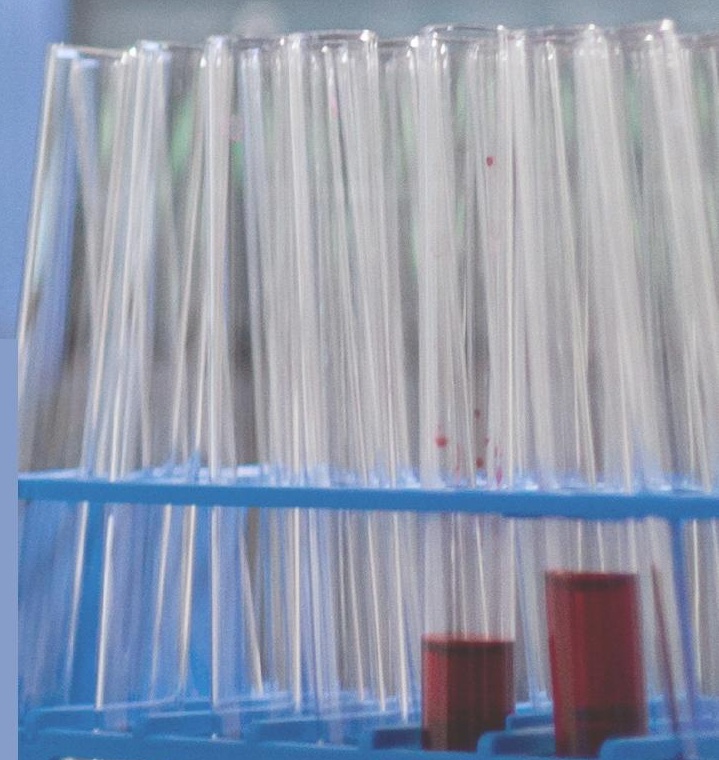

\section{EPIDEMIOLOGIA}

\section{Volume 2}

\section{Organizadora:}

Pauliana Valéria Machado Galvão 


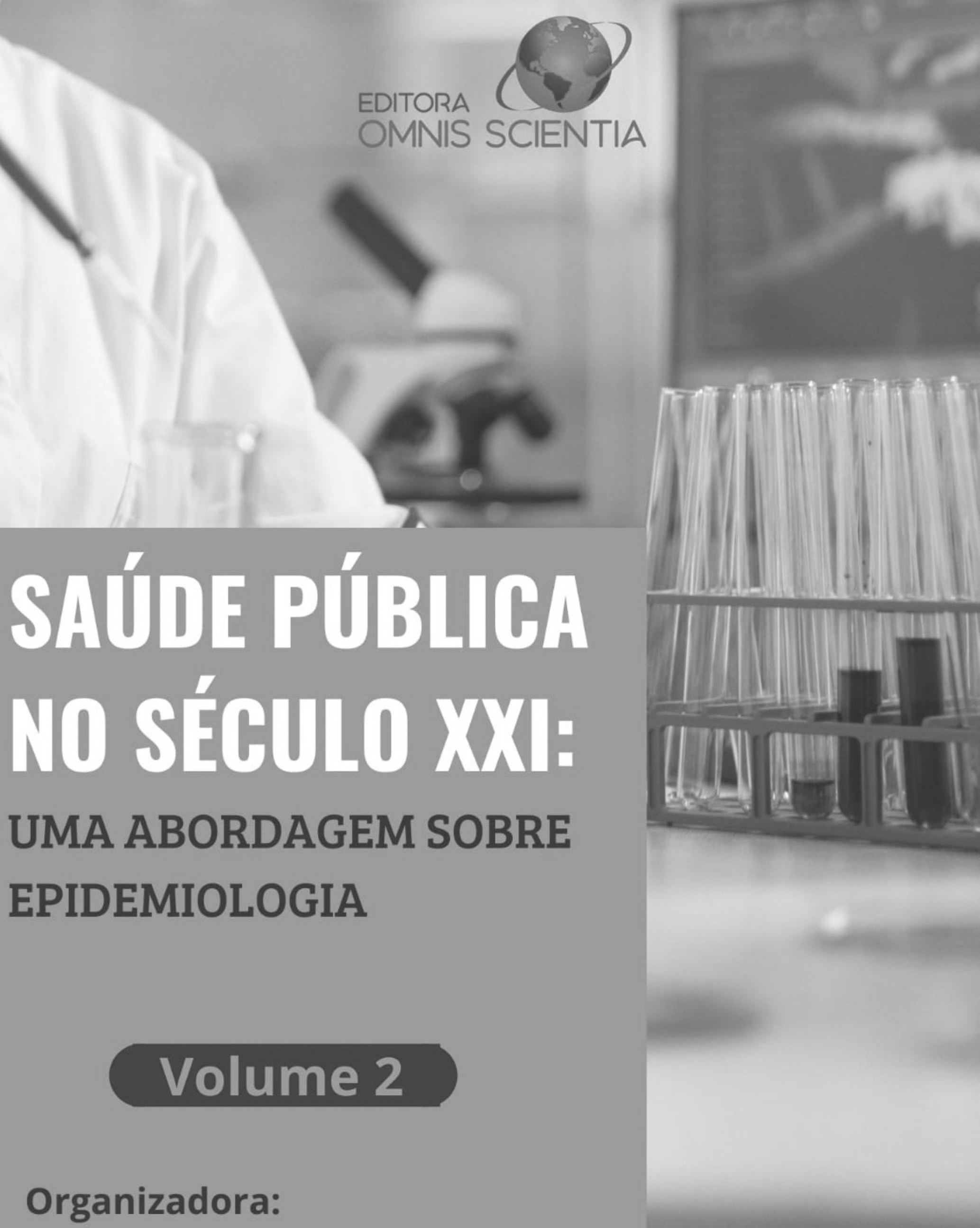

Pauliana Valéria Machado Galvão 
Editora Omnis Scientia

SAÚDE PÚBLICA NO SÉCULO XXI:

UMA ABORDAGEM SOBRE EPIDEMIOLOGIA

Volume 2

$2^{\mathrm{a}}$ Edição

TRIUNFO - PE

2021 


\section{Editor-Chefe}

Me. Daniel Luís Viana Cruz

\section{Organizadora}

Dra. Pauliana Valéria Machado Galvão

\section{Conselho Editorial}

Dr. Cássio Brancaleone

Dr. Marcelo Luiz Bezerra da Silva

Dra. Pauliana Valéria Machado Galvão

Dr. Plínio Pereira Gomes Júnior

Dr. Walter Santos Evangelista Júnior

Dr. Wendel José Teles Pontes

Editores de Área - Ciências da Saúde

Dra. Camyla Rocha de Carvalho Guedine

Dra. Cristieli Sérgio de Menezes Oliveira

Dr. Leandro dos Santos

Dr. Hugo Barbosa do Nascimento

Dr. Marcio Luiz Lima Taga

Dra. Pauliana Valéria Machado Galvão

Assistente Editorial

Thialla Larangeira Amorim

Imagem de Capa

Freepik

\section{Edição de Arte}

Vileide Vitória Larangeira Amorim

\section{Revisão}

Os autores

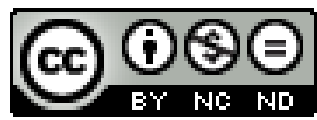

Este trabalho está licenciado com uma Licença Creative Commons - AtribuiçãoNãoComercial-SemDerivações 4.0 Internacional.

O conteúdo abordado nos artigos, seus dados em sua forma, correção e confiabilidade são de responsabilidade exclusiva dos autores. 


\section{Dados Internacionais de Catalogação na Publicação (CIP) (eDOC BRASIL, Belo Horizonte/MG)}

S255 Saúde pública no século XXI [livro eletrônico] : uma abordagem sobre epidemiologia: volume 2 / Organizadora Pauliana Valéria Machado Galvão. - Triunfo, PE: Omnis Scientia, 2021. $121 \mathrm{p}$.

Formato: PDF

Requisitos de sistema: Adobe Acrobat Reader Modo de acesso: World Wide Web Inclui bibliografia ISBN 978-65-88958-69-8

DOI 10.47094/978-65-88958-69-8

1. Epidemiologia. 2. Política de saúde - Brasil. 3. Saúde pública. I. Galvão, Pauliana Valéria Machado.

CDD 614.4

Elaborado por Maurício Amormino Júnior - CRB6/2422

Editora Omnis Scientia

Triunfo - Pernambuco - Brasil

Telefone: +55 (87) 99656-3565

editoraomnisscientia.com.br

contato@editoraomnisscientia.com.br 


\section{PREFÁCIO}

A Epidemiologia permite a identificação do processo saúde-doença sob um ponto de vista coletivo e sua consolidação tende a otimizar os esforços de gestão e planejamento do uso dos recursos em saúde.

A atualidade vivida sob a ótica de uma pandemia deve fortalecer a importância da Epidemiologia como abordagem científica essencial para o desenvolvimento de uma saúde pública de qualidade e trazer luz a diversas abordagens epidemiológicas tende a influenciar e incentivar a ampliação de outros estudos no formato. E o segundo volume do livro Saúde Pública no Século XXI: uma abordagem sobre a Epidemiologia vem reforçar este compromisso iniciado no primeiro volume. Este livro contribui para fortalecer os pesquisadores da área e trazer uma vitrine à potencialidade de trabalhos a serem desenvolvidos e abordou diversos problemas muito importantes para a Saúde Pública: COVID, mortalidade materna, doenças cardiovasculares, hanseníase, pacientes submetidos a hemotransfusão e desafios na condução da sífilis congênita.

Em nossos livros selecionamos um dos capítulos para premiação como forma de incentivo para os autores, e entre os excelentes trabalhos selecionados para compor este livro, o premiado foi o capítulo 1, intitulado "AVALIAÇÃO DA MORTALIDADE MATERNA A $5^{\circ}$ REGIONAL DE SAÚDE DO ESTADO DO PARANÁ: SÉRIE TEMPORAL”. 


\section{SUMÁRIO}

CAPÍTULO 1.

AVALIAÇÃO DA MORTALIDADE MATERNA A $5^{\circ}$ REGIONAL DE SAÚDE DO ESTADO DO PARANÁ: SÉRIE TEMPORAL

Dannyele Cristina da Silva

Giovana Frazon de Andrade

Elaine Maria Rodrigues

Leticia Gramazio Soares

Raiane Maria Rocha Pinheiro

Stefany Luana de Oliveira

Thais Amanda Rossa

DOI: 10.47094/978-65-88958-69-8/11-22

CAPÍTULO 2

PERFIL EPIDEMiológico E AVALIAÇÃO DAS POLÍticas PÚBLICAS DE ENFRENTAMENTO À MORTALIDADE MATERNA NO ESTADO DO AMAPÁ ENTRE OS ANOS DE 2014 E 2019

Edson Fábio Brito Ribeiro

Giovana Carvalho Alves

Lucas Facco Silva

Gustavo Aurélio Linhares de Magalhães

Tamires Barbosa da Silva

Maria Helena Mendonça de Araújo

Silvia Claudia Cunha Maues

Rosilene Ferreira Cardoso

DOI: 10.47094/978-65-88958-69-8/23-38 
GRAU DE INCAPACIDADE DOS PACIENTES COM HANSENÍASE EM UM MUNICÍPIO HIPERENDÊMICO: DO DIAGNÓSTICO A ALTA

Danielle Conceição de Barros Costa Valério

Josiele Gomes de Oliveira

Letícia Silveira Goulart

Lorena Araújo Ribeiro Gonçalves

Ricardo Alves de Olinda

Débora Aparecida da Silva Santos

DOI: $10.47094 / 978-65-88958-69-8 / 39-56$

CAPÍTULO 4 .57

ANÁLISE TRANSVERSAL DOS DADOS DA COVID-19 EM MINAS GERAIS: A IMPORTÂNCIA DA EPIDEMIOLOGIA NO CONTEXTO DE CRISE

Amanda Menezes Oliveira

Vitória da Silva Marques

Ana Paula de Lima Bezerra

Isadora Oliveira Gondim

Franciele Carolina Barbosa

Luyller Bruno Esteves de Souza

Virgínia Fernanda Alves

Fernanda Gonçalves de Souza

DOI: 10.47094/978-65-88958-69-8/57-68

CAPÍTULO 5. .69

COVID-19, SAÚDE MENTAL E USO DE SUBSTÂNCIAS: DADOS EPIDEMIOLÓGICOS, RISCOS ASSOCIADOS E NOVAS INTERVENÇÕES

Richard Alecsander Reichert

Beatriz de Oliveira Lavezzo

Thaís Hoffmann Stump

Beatriz Iannotta

Wanderlei Abadio de Oliveira 
Denise de Micheli

Adriana Scatena

Felipe Anselmo Pereira

Rosana Fanucci Silva Ramos

Suzanna Araújo Preuhs

Gabriella Di Girolamo Martins

André Luiz Monezi Andrade

DOI: 10.47094/978-65-88958-69-8/69-88

CAPÍtULO 6. .89

DOENÇAS CARDIOVASCULARES RELACIONADAS AO TRABALHO: DESAFIOS PARA O ESTABELECIMENTO DO NEXO CAUSAL

Regina de Souza Moreira

Jorgana Fernanda de Souza Soares

DOI: 10.47094/978-65-88958-69-8/89-97

CAPÍTULO 7. .98

DESAFIOS DA EQUIPE DE ENFERMAGEM NA PREVENÇÃO DA SÍFILIS CONGÊNITA NA ATENÇÃO PRIMÁRIA: REVISÃO DE LITERATURA

Janaína Dahmer

Wuelison Lelis de Oliveira

Ianaê Gomes dos Santos

Cinthia Tayná Gouveia Brito

Laryssa Rodrigues Carvalho de La Torre

Alciele do Nascimento Soares

Bianca Caroline Bianchetto

Daniele Roecker Chagas

Flaviane Cristina da Silva

Gilvan Salvador Júnior

Loiane Claire Bianqui

Ruan Felipe Rego de Souza 
CAPÍTULO 8...

PERFIL CLÍNICO E EPIDEMIOLÓGICO DE PACIENTES PÓS-CIRÚRGICOS SUBMETIDOS À HEMOTRANSFUSÃO

Lidyane Rodrigues Oliveira Santos

Leticia Oliveira Cruz

Pamela Nayara dos Santos Marques

Kelson Antonio de Oliveira Santos

Maria Tamires Alves Ferreira

Talvany Luís de Barros

Grazielle Roberta Freitas da Silva

Ingryd Lannay de Carvalho Silva

Adriana de Sousa Mourão

Aline Borges de Araújo

Louise de Macedo Sousa Frazão

Paula Fernanda Lemos Veras

DOI: 10.47094/978-65-88958-69-8/105-117 


\section{CAPÍTULO 3}

\section{GRAU DE INCAPACIDADE DOS PACIENTES COM HANSENÍASE EM UM \\ MUNICÍPIO HIPERENDÊMICO: DO DIAGNÓSTICO A ALTA}

\section{Danielle Conceição de Barros Costa Valério;}

Universidade Federal de Rondonópolis (UFR), Rondonópolis, Mato Grosso.

https://orcid.org/0000-0002-9412-8325

\section{Josiele Gomes de Oliveira²;}

Universidade Federal de Rondonópolis (UFR), Rondonópolis, Mato Grosso.

https://orcid.org/0000-0003-3266-1391

\section{Letícia Silveira Goulart³;}

Universidade Federal de Rondonópolis (UFR), Rondonópolis, Mato Grosso.

http://orcid.org/0000-0003-1452-4908

\section{Lorena Araújo Ribeiro Gonçalves ${ }^{4}$;}

Universidade Federal de Rondonópolis (UFR), Rondonópolis, Mato Grosso.

https://orcid.org/ 0000-0003-0525-4758

\section{Ricardo Alves de Olinda ${ }^{5}$;}

Universidade Estadual da Paraíba (UEPB), Rondonópolis, Paraíba.

https://orcid.org/0000-0002-0509-8428

Débora Aparecida da Silva Santos ${ }^{6}$.

Universidade Federal de Rondonópolis (UFR), Rondonópolis, Mato Grosso.

https://orcid.org/0000-0003-1862-7883

RESUMO: A hanseníase quando não diagnosticada precocemente, pode gerar incapacidades físicas ou sequelas permanentes. Este estudo objetivou comparar o grau de incapacidade física e as deficiências presentes no diagnóstico e na alta ambulatorial em pacientes com hanseníase. Estudo de caráter descritivo, retrospectivo, fonte documental com delineamento quantitativo. A amostra foi composta por todos pacientes que realizaram tratamento de hanseníase no Serviço de Atenção Especializada de Rondonópolis (MT) entre 2017 a 2018. As variáveis observadas foram os dados sociodemográficos, clínico epidemiológicos e avaliação neurológica simplificada, que inclui avaliação dos graus de incapacidade física (GIF). Foi utilizada estatística descritiva, os testes aderência de Quiquadrado e Exato de Fisher considerando nível de significância de 5\% e de McNemar. A pesquisa foi aprovada pelo Comitê de Ética em Pesquisa (CAEE 97441618.2.0000.8088). Foram analisados 72 
casos de hanseníase com GIF, sendo que a maioria não houve alterações no diagnóstico $(73,1 \%)$ e alta $(84,72 \%)$. Houve significância estatística do número de casos com GIF e nas variáveis diminuição da sensibilidade córnea e catarata, nervo ulnar e mediano, avaliação de força ao abrir o dedo mínimo e elevar o polegar e na avaliação fibular. Neste sentido, medidas preventivas voltadas às incapacidades são essenciais e devem ser intensificadas no tratamento destes usuários.

PALAVRAS-CHAVE: Hanseníase. Pessoas com incapacidade. Degeneração neural. Epidemiologia.

\title{
DEGREE OF DISABILITY OF LEPROSY PATIENTS IN A HYPER-ENDEMIC
}

\section{MUNICIPALITY: FROM DIAGNOSIS TO DISCHARGE}

\begin{abstract}
Leprosy, when not diagnosed early, can generate physical disabilities or permanent sequelae. This study aimed to compare the degree of physical incapacity and the deficiencies present at diagnosis and at outpatient discharge in patients with leprosy. Descriptive, retrospective study, documental source with quantitative design. The sample consisted of all patients who underwent treatment for leprosy at the Specialized Care Service of Rondonópolis (MT) between 2017 and 2018. The variables observed were sociodemographic, clinical epidemiological and simplified neurological assessment data, which includes assessment of the degrees of physical disability (GIF). Descriptive statistics, Chi-square and Fisher's exact adherence tests were used, considering a significance level of 5\% and McNemar. The research was approved by the Research Ethics Committee (CAEE 97441618.2.0000.8088). Seventy-two cases of leprosy with GIF were analyzed, most of which had no changes in diagnosis $(73.1 \%)$ and discharge $(84.72 \%)$. There was statistical significance in the number of cases with GIF and in the variables decreased corneal sensitivity and cataracts, ulnar and median nerves, strength assessment when opening the little finger and lifting the thumb and in the peroneal assessment. In this sense, preventive measures aimed at disabilities are essential and should be intensified in the treatment of these users.
\end{abstract}

KEY-WORDS: Leprosy. People with disabilities. Neural degeneration. Epidemiology.

\section{INTRODUÇÃO}

A hanseníase é uma doença infectocontagiosa, cujo agente etiológico é o Mycobacterium leprae. A doença tem afinidade com os nervos superficiais da pele e troncos nervosos periféricos com alta cronicidade, podendo afetar os olhos e órgãos internos. Quando não diagnosticada precocemente desenvolve alguma alteração no grau de incapacidade ou leva a sequelas permanentes (BRASIL, 2017).

Determinados sinais e sintomas estão presentes na hanseníase, como manchas avermelhadas ou amarronzadas que se apresentam em qualquer região do corpo. Essas lesões geralmente provocam a perda ou alterações de sensibilidades térmica, tátil e dolorosa, podendo comprometer a diminuição dos pêlos e do suor. Outros sinais que podem ocorrer são a presença de sensações de dor, choque, 
formigamento, fisgado e agulhado ao longo dos nervos dos braços e das pernas (BRASIL, 2019).

Geralmente, as alterações de capacidades da hanseníase são causadas pelas vias inflamatórias e neurogênicas, que podem ser avaliadas como deficiências primárias e secundárias. Estas levam ao comprometimento de tecidos e órgãos, atingindo regiões como face, membros superiores e inferiores e, conforme o grau de incapacidade, evoluir para a formação de garras rígidas, mal perfurante plantar e reabsorções ósseas (BRASIL, 2008).

Conforme o Ministério da Saúde (MS), as incapacidades físicas podem estar presentes em qualquer forma clínica, devendo ser avaliadas durante o diagnóstico no início e no fim do tratamento. $\mathrm{O}$ tratamento de hanseníase tem como complemento fundamental a prevenção das incapacidades, no intuito de haver a continuidade de todas as atividades sociais e profissionais do indivíduo durante e depois do tratamento poliquimioterápico (BRASIL, 2004; SILVA SOBRINHO et al., 2007).

Durante o tratamento da doença é fundamental que ocorra a avaliação neurológica no diagnóstico, na reação e na alta por cura. Utiliza-se um instrumento chamado de Avaliação Neurológica Simplificada, fazendo parte da avaliação à análise através da inspeção, palpação/percussão e avaliação funcional dos nervos acometidos, onde é feita a análise da sensibilidade e força muscular, com isso é classificado o grau de incapacidade física (BRASIL, 2017).

Existe uma classificação quanto ao número de lesões, carga bacilare o nível de comprometimento dos nervos da região periférica, dos pacientes com a hanseníase. Quando avaliado as complicações, o GIF 0 é atribuído quando não ocorre o comprometimento neural, no grau 1 acontece a diminuição ou a perda da sensibilidade dos olhos, mãos e pés e no grau 2 há a presença de lesões mais graves nos olhos, mãos e pés (RIBEIRO; LANA, 2015).

As sequelas desenvolvidas pela hanseníase geram uma série de agravos aos acometidos pela doença, devido ao comprometimento de nervos responsáveis por respostas a dor, visão e sensibilidade tátil. Além de sequelas permanentes, estão mais suscetíveis a acidentes como: feridas, queimaduras, fraturas até amputações. Como consequência, as sequelas psicológicas e o distanciamento social acompanham o paciente intervindo na sua qualidade de vida (BRASIL, 2017).

Conforme a Organização Mundial de Saúde (OMS), em 2018, foram detectados 208.619 casos novos de hanseníase, sendo que 30.957 casos foram na região das Américas e 28.660 (92,6\% do total das Américas) estão no Brasil. Apesar dos avanços adquiridos nos últimos anos, o país continua entre os 22 países com as mais altas cargas da doença em nível global, detendo 92\% do total dos casos dos países das América e mantendo a segunda posição na detecção de casos novos em relação a avaliação do Grau de Incapacidade Física (GIF), dos 24.780 (86,5) analisados no diagnóstico, apresentaram alterações físicas visíveis com 2.109 com grau de incapacidade física 2 representando $(8,5 \%)$, o que faz com que o Brasil esteja em segundo lugar com maior carga da doença, estando atrás somente da Índia (OMS, 2019).

Segundo o Boletim Epidemiológico 2020, nos anos de 2014 a 2018, foram diagnosticados no Brasil 140.578 casos novos de hanseníase. Nesse período a taxa média de detecção foi de 13,64 casos novos para 100 mil. Avaliando os indicadores das macrorregiões, Mato Grosso ficou em segundo 
lugar, com 62,08 casos por 100 mil habitantes (BRASIL, 2020).

Observa-se que Mato Grosso possui a maior taxa de notificações do país, sendo que a detecção da hanseníase atinge regiões específicas, pois há uma carência nas ofertas dos serviços de saúde, refletindo num diagnóstico tardio e com presença de incapacidade. A presença de problemas com relação ao manejo clínico e tratamento da doença, bem como as falhas nos serviços de reabilitação e pouca participação da sociedade referente ao preconceito e o estigma da doença, acabam prejudicando a detecção precoce da hanseníase e sua efetividade do tratamento, pois muitas vezes os usuários já possuem alguma incapacidade (MATO GROSSO, 2018).

Conforme dados do boletim epidemiológico 2020, entre os anos de 2009 a 2018 no Brasil foram diagnosticados 20.785 casos novos de hanseníase que apresentaram alteração de incapacidade física Grau 2, que é reflexo da taxa de detecção geral de casos novos da doença. Conforme o documento foi observado que a taxa no início do estudo com relação ao GIF 2 foi de 12,72 e em 2018 de 10,08 casos por 1 milhão de habitantes, sendo minimizada em $21 \%$. No entanto, somente na região CentroOeste houve um discreto aumento, com uma taxa de GIF 2 de 20,87 com uma taxa elevada de 21,63 casos por 1 milhão de habitantes. Esses dados são preocupantes, visto que é possível identificar a presença de um diagnóstico tardio, levando um maior comprometimento físico dos usuários devido às alterações provocadas pela hanseníase (BRASIL, 2020).

Partindo do pressuposto que Rondonópolis é um município hiperendêmico para hanseníase e os casos quando diagnosticados podem apresentar algum grau de incapacidade, justifica-se a relevância da pesquisa, a fim de contribuir para o diagnóstico precoce da doença, melhorar a avaliação dos graus de incapacidades, prevenindo as sequelas e promovendo o autocuidado dos usuários.

Desta forma, o objetivo deste estudo foi comparar o grau de incapacidade física e as deficiências presentes no diagnóstico e na alta ambulatorial em pacientes com hanseníase diagnosticados no Serviço de Atenção Especializada em Rondonópolis (MT) entre 2017 a 2018.

\section{METODOLOGIA}

Trata-se de um estudo de caráter descritivo, retrospectivo, e fonte documental com delineamento quantitativo. A coleta de dados compreendeu o período entre 2017 a 2018 dos pacientes que realizaram tratamento no Serviço de Atenção Especializada (SAE) do município de Rondonópolis, Mato Grosso. (MT).

O cenário do estudo foi o SAE DST, HIV e Hepatites cadastrado no dia 19/12/2009, registrado no Cadastro Nacional dos Estabelecimentos de Saúde (CNES) nº 6392946, é um tipo de estabelecimento para apoio, diagnóstico e terapia. Caracterizado como uma unidade conveniada ao Sistema Único de Saúde (SUS) com atividade ambulatorial, busca a promoção da saúde, prevenção de doenças e agravos na produção do cuidado, entre elas, a hanseníase.

Os dados do estudo foram compostos por 186 prontuários de pacientes diagnosticados com hanseníase no SAE. Para o delineamento da amostra, foram instituídos os seguintes critérios de inclusão: pacientes cujos prontuários possuíam datas de início e finalização do tratamento para 
hanseníase no centro de referência e que possuíam preenchida a ficha de avaliação simplificada das funções neurais e complicações no diagnóstico e na alta por cura. Quanto aos critérios de exclusão foram: os prontuários de pacientes com comprometimento cognitivo; informações incompletas relacionadas a avaliação simplificada das funções neurais e complicações e determinação do GIF e os pacientes que não realizaram o tratamento no tempo preconizado pelo Ministério da Saúde (MS). Depois de compilados os dados, a amostra estudada foi composta por 72 usuários.

A amostra ocorreu através de um instrumento estruturado aplicado às fichas de notificação compulsória e de avaliação neurológica simplificada. As variáveis observadas foram dados sociodemográficos, clínico epidemiológicos e avaliação neurológica simplificada, que inclui avaliação dos graus de incapacidade física (GIF). Os pacientes foram avaliados por enfermeiros e terapeuta ocupacional com experiência em avaliação dermatoneurológica, treinados de modo a haver uma uniformização da técnica. Para esta avaliação é utilizado o protocolo nacional de avaliação neurológica simplificada e a classificação do grau de incapacidade conforme os parâmetros adotados pelo MS; ambos registrados nos formulários citados.

As variáveis coletadas da ficha de notificação foram: data da notificação, data do diagnóstico, data do início da Poliquimioterapia (PQT), forma clínica, idade, sexo, ocupação, bairro, zona territorial, cor, gestante, escolaridade, modo de entrada, modo de detecção, baciloscopia, classificação operacional, esquema terapêutico inicial.

Do formulário para avaliação neurológica simplificada, os dados foram compilados com base nas seguintes informações: data da alta; data da alta da PQT; avaliação da região do nariz (queixa principal, ressecamento, ferida e perfusão de septo); região dos olhos (queixa principal, fecha olhos com e sem força, triquíase, ectrópio, diminuição da sensibilidade da córnea, opacidade córnea e catarata); região dos membros superiores (queixa principal, avaliação dos nervos ulnar, mediano e radial); avaliação da força dos membros superiores (abrir dedo mínimo, abdução do $5^{\circ}$ dedo - nervo ulnar, elevar polegar, abdução do polegar); nervo mediano (elevar o punho, extensão de punho e nervo radial); membros inferiores (queixa principal, palpação dos nervos fibular e tibial posterior) e avaliação da força do membros inferiores (elevar o hálux, extensão de hálux, elevar o pé e dorsiflexão do pé). Por fim, as variáveis com relação a classificação do grau de incapacidade incluíram os seguintes dados: avaliação dos olhos, mãos, pés e maior grau de incapacidade que foram realizadas no diagnóstico e alta do tratamento.

Cabe ressaltar que no formulário para avaliação neurológica simplificada, o GIF varia de zero (0) até dois (2). Quando o valor é considerado zero (0) significa que não possui dano presente nos olhos, mãos e pés; já o grau um (1) indica diminuição ou perda da sensibilidade nessas regiões e no grau dois (2) ocorre a presença de deformidades que estão visíveis decorrente da hanseníase. A avaliação do GIF conforme o MS deve ser realizada no diagnóstico, durante reações e na alta por cura (BRASIL, 2016).

Para a análise de dados foi utilizada a estatística descritiva, utilizando frequências simples, absolutas e percentuais para as variáveis categóricas e organização dos resultados em tabelas com as respectivas distribuições de frequências. Na sequência, foi aplicado o teste de aderência de qui- 
quadrado para verificar a adequabilidade do modelo probabilístico aos dados da pesquisa.

Ademais, para verificar possíveis associações entre variáveis dependente e independentes, foram utilizados o teste Qui-quadrado e o teste Exato de Fisher nos casos onde as frequências esperadas foram menores que 5 , considerando o nível de significância de $5 \%(p<0,005)$. Foi aplicado o teste de McNemar para analisar frequências (proporções) duas a duas, caso contrário, foi utilizado o teste McNemar- Bowker. As análises foram realizadas com o auxílio do software estatístico R ( $\mathrm{R}$ CORE TEAM, 2020).

A pesquisa seguiu os preceitos éticos em pesquisa de acordo com a Resolução CNS n ${ }^{\circ} 466$ de 12 de dezembro de 2012 (BRASIL, 2012) do Conselho Nacional de Saúde do Ministério da Saúde para pesquisa em seres humanos, sendo aprovado pelo Comitê de Ética e Pesquisa da Universidade Federal de Mato Grosso, campus Rondonópolis, CAEE número 97441618.2.0000.8088.

\section{RESULTADOS}

Neste estudo foram analisados 72 casos novos de hanseníase com GIF notificados nos anos de 2017 a 2018. Na maioria dos casos não houveram alterações na capacidade física durante o diagnóstico $(73,1 \%)$ e alta $(84,72 \%)$. Houve significância estatística do número de casos com GIF tanto no diagnóstico como na alta (Tabela 1).

Tabela 1. Distribuição dos casos de hanseníase com Grau de Incapacidade Física (GIF) no diagnóstico e alta dos anos 2017 e 2018.

\begin{tabular}{|c|c|c|c|c|c|c|c|}
\hline Grau & IncapacidadeFísica (GIF) & Dia & & & Alta & & \\
\hline & & $\mathbf{N}$ & $\%$ & Valor p & $\mathbf{N}$ & $\%$ & Valor p \\
\hline Grau 0 & & 53 & 73,61 & $<\mathbf{0 , 0 0 1}$ & 61 & 84,72 & $<0,001$ \\
\hline Grau 1 & & 14 & 19,44 & & 7 & 9,72 & \\
\hline Grau 2 & & 4 & 5,56 & & 4 & 5,56 & \\
\hline Não avaliado & & 1 & 1,39 & & 0 & 0 & \\
\hline Total & & 72 & 100 & & 72 & 100 & \\
\hline
\end{tabular}

Fonte: Dados da pesquisa. Elaborada pelos autores.

O perfil sociodemográfico dos casos de hanseníase com GIF entre os anos de 2017 e 2018, foi: sexo masculino (51,39\%), faixa etária de 16 a 59 anos (65,28\%), profissional liberal (52,78\%), ensino fundamental $(54,17 \%)$, zona urbana $(90,28 \%)$ e cor parda $(63,89 \%)$. As variáveis que apresentaram significância foram faixa etária, ocupação, escolaridade, zona de residência e cor (Tabela 2).

O perfil clínico epidemiológico dos casos de hanseníase entre os anos de 2017 e 2018 foi: caso novo $(98,61 \%)$, modo de detecção por encaminhamento $(56,94 \%)$, baciloscopia não realizada (97,22\%), classificação operacional multibacilar (95,83\%), esquema terapêutica inicial PQT/MB 12 meses $(95,83 \%)$, forma clínica Dimorfa $(87,50 \%)$. Todas estas variáveis foram significativas para os GIF dos casos de hanseníase estudados (Tabela 2). 
Tabela 2: Perfil sociodemográfico e perfil clínico epidemiológico dos casos de hanseníase com Grau de Incapacidade Física (GIF) dos anos 2017 e 2018.

\begin{tabular}{|c|c|c|c|c|c|c|c|c|c|}
\hline \multirow{2}{*}{$\begin{array}{l}\text { Variáveis } \\
\text { Sexo }\end{array}$} & \multirow{2}{*}{\multicolumn{2}{|c|}{$\mathbf{N}$}} & \multirow[t]{2}{*}{$\%$} & \multirow[t]{2}{*}{ Valor $\mathbf{p} V$} & Variáveis & \multirow[t]{2}{*}{$\mathbf{N}$} & \multicolumn{2}{|c|}{$\%$} & \multirow[t]{2}{*}{ Valor $\mathbf{p}$} \\
\hline & & & & & Modo de entrada & & & & \\
\hline Feminino & 35 & & 48,61 & 0,8137 & Caso Novo & & 71 & 98,61 & $<0,001$ \\
\hline Masculino & 37 & & 51,39 & & Transferência do mesmc & nicípio & 1 & 1,39 & \\
\hline Faixa Etária & & & & & Modo de detecção & & & & \\
\hline$\leq 15$ anos & 3 & & 4,17 & $<0,001$ & Encaminhamento & & 41 & 56,94 & $<0,001$ \\
\hline 16 a 59 anos & 47 & & 65,28 & & Demanda Espontânea & & 27 & 37,50 & \\
\hline \multirow[t]{2}{*}{$\geq 60$ anos } & 22 & & 30,56 & & Exame de Coletividade & & 1 & 1,39 & \\
\hline & & & & & Exame de Contatos & & 3 & 4,17 & \\
\hline Ocupação & & & & & Baciloscopia & & & & \\
\hline Profissional & & & & $<\mathbf{0 , 0 0 1}$ & Negativa & & & & $<\mathbf{0 , 0 0 1}$ \\
\hline Liberal & 38 & & 52,78 & & & & 1 & 1,39 & \\
\hline Aposentado/ & 11 & & 15,28 & & & & 70 & 97,22 & \\
\hline Pensionista & & & & & & & & & \\
\hline Estudante & 6 & & 8,33 & & Em branco / Ignorado & & 1 & 1,39 & - \\
\hline \multirow[t]{2}{*}{ Do Lar } & 17 & & 23,61 & & & & & & \\
\hline & & & & & Classificação & & & & \\
\hline Escolaridade & & & & & Operacional & & & & \\
\hline Analfabeto & & 8 & 11,11 & $<0,001$ & Paucibacilar & & 3 & 4,17 & $<0,001$ \\
\hline \multicolumn{10}{|l|}{ Até ensino fundamental } \\
\hline & & 39 & 54,17 & & Multibacilar & & 69 & 95,83 & \\
\hline \multicolumn{10}{|l|}{ Ensino médio completo } \\
\hline \multicolumn{10}{|l|}{ ou } \\
\hline incompleto & 14 & & 19,44 & & & & & & \\
\hline Superior completo & & & & & EsquemaTerapêutico & & & & \\
\hline ou incompleto & 8 & & 11,11 & & & & & & \\
\hline Em branco / & & & & - & & & & & $<0,001$ \\
\hline \multirow[t]{2}{*}{ Ignorado } & 3 & & 4,17 & & PQT/PB 6 Doses & & 3 & 4,17 & \\
\hline & & & & & PQT/MB/ 12 Meses & & 69 & 95,83 & \\
\hline Zona & & & & & Forma Clínica & & & & \\
\hline Urbana & 65 & & 90,28 & $<\mathbf{0 , 0 0 1}$ & Tuberculóide & & 8 & 11,11 & \\
\hline \multirow[t]{2}{*}{ Rural } & 7 & & 9,72 & & Dimorfa & & 63 & 87,50 & \\
\hline & & & & & Virchowiana & & 1 & 1,39 & \\
\hline \multicolumn{10}{|l|}{ Cor } \\
\hline Branca & 14 & & 19,44 & $<\mathbf{0 , 0 0 1}$ & & & & & \\
\hline Preta & 10 & & 13,89 & & & & & & \\
\hline Parda & & 46 & 63,89 & & & & & & \\
\hline \multirow[t]{2}{*}{ Em branco / Ignorado } & & & & - & & & & & \\
\hline & & 2 & 2,78 & & & & & & \\
\hline
\end{tabular}

Fonte: Dados da pesquisa. Elaborada pelos autores.

\section{SAÚDE PÚBLICA NO SÉCULO XXI:}


A avaliação da face no diagnóstico na região do nariz foi: sem queixas $(95,83 \%)$, não teve ressecamento (100\%), não teve ferida (100\%), não teve perfusão de septo (100\%). Região dos olhos: sem queixas olho direito $(76,39 \%)$ e olho esquerdo $(77,78 \%)$, fecha os olhos sem força $(69,44 \%)$, fecha os olhos com força (66,67\%), triquíase e ectrópio sem alteração em ambos os lados (75\%), diminuição da sensibilidade córnea sem alteração direito $(98,61 \%)$ e esquerdo $(95,83)$, opacidade córnea sem alteração $(98,61 \%)$, catarata sem alterações direito $(97,22 \%)$ e esquerdo $(95,83 \%)$ (Tabela $3)$.

A avaliação da face durante a alta na região do nariz: sem queixas (95,83\%), não teve ressecamento (100\%), não teve ferida (100\%), não teve perfusão de septo (100\%). Região dos olhos: sem queixas $(86,11 \%)$, fecha os olhos sem força $(94,44 \%)$, fecha os olhos com força $(93,06 \%)$, triquíase e ectrópio sem alteração $(95,83 \%)$, diminuição da sensibilidade córnea sem alteração (100\%), opacidade córnea sem alteração (100\%), catarata sem alterações (95,83\%) (Tabela 3). As variáveis com significância estatística foram diminuição da sensibilidade córnea durante o diagnóstico e catarata durante o diagnóstico e a alta (Tabela 3).

Tabela 3. Comparação da avaliação da face durante o diagnóstico e alta dos casos de hanseníase com Grau de Incapacidade Física (GIF) dos anos 2017 e 2018. Rondonópolis (MT), 2020.

\begin{tabular}{|c|c|c|c|c|c|c|c|c|c|c|}
\hline & \multirow{3}{*}{$\begin{array}{c}\text { Direito } \\
\mathbf{N}\end{array}$} & \multicolumn{4}{|c|}{ Diagnóstico } & \multicolumn{4}{|c|}{ Alta } & \multirow[b]{3}{*}{ Valor p } \\
\hline & & & \multicolumn{3}{|c|}{ Esquerdo } & \multicolumn{2}{|c|}{ Direito } & \multicolumn{2}{|c|}{ Esquerdo } & \\
\hline & & $\%$ & $\mathbf{N}$ & $\%$ & Valor $\mathbf{p}$ & $\mathbf{N}$ & $\%$ & $\mathbf{N}$ & $\%$ & \\
\hline \multicolumn{11}{|l|}{ NARIZ } \\
\hline \multicolumn{11}{|l|}{ Queixa principal } \\
\hline Sem queixas & 69 & 95,83 & 69 & 95,83 & 0,9999 & 69 & 95,83 & 69 & 95,83 & 0,9999 \\
\hline Dor & 1 & 1,39 & 1 & 1,39 & & 1 & 1,39 & 1 & 1,39 & \\
\hline Formigamento & 2 & 2,78 & 2 & 2,78 & & 2 & 2,78 & 2 & 2,78 & \\
\hline \multicolumn{11}{|l|}{ Ressecamento } \\
\hline Sim & 0 & 0 & 0 & 0 & - & 0 & 0 & 0 & 0 & - \\
\hline Não & 72 & 100 & 72 & 100 & & 72 & 100 & 72 & 100 & \\
\hline \multicolumn{11}{|l|}{ Ferida } \\
\hline Sim & 0 & 0 & 0 & 0 & - & 0 & 0 & 0 & 0 & - \\
\hline Não & 72 & 100 & 72 & 100 & & 72 & 100 & 72 & 100 & \\
\hline \multicolumn{11}{|l|}{ Perfusão de Septo } \\
\hline Sim & 0 & 0 & 0 & 0 & - & 0 & 0 & 0 & 0 & - \\
\hline Não & 72 & 100 & 72 & 100 & & 72 & 100 & 72 & 100 & \\
\hline \multicolumn{11}{|l|}{ OLHOS } \\
\hline \multicolumn{11}{|l|}{ Queixa principal } \\
\hline Sem queixas & 55 & 76,39 & 56 & 77,78 & 0,9876 & 62 & 86,11 & 62 & 86,11 & 0,9999 \\
\hline Dor & 1 & 1,39 & 1 & 1,39 & & 1 & 1,39 & 1 & 1,39 & \\
\hline Alteração de Visão & 16 & 22,22 & 15 & 20,83 & & 9 & 12,50 & 9 & 12,50 & \\
\hline \multicolumn{11}{|l|}{ Fecha os olhos sem } \\
\hline $\begin{array}{l}\text { força } \\
\text { Sim }\end{array}$ & 50 & 69,44 & 50 & 69,44 & 0,9999 & 68 & 94,44 & 68 & 94,44 & 0,9999 \\
\hline Não & 22 & 30,56 & 22 & 30,56 & & 4 & 5,56 & 4 & 5,56 & \\
\hline
\end{tabular}

Fecha os olhos com força 


\begin{tabular}{|c|c|c|c|c|c|c|c|c|c|c|c|}
\hline $\operatorname{Sim}$ & & 48 & 66,67 & 48 & 66,67 & 0,9999 & 67 & 93,06 & 67 & 93,06 & 0,9999 \\
\hline Não & & 24 & 33,33 & 24 & 33,33 & & 4 & 5,56 & 4 & 5,56 & \\
\hline Não avaliado & & 0 & 0 & 0 & 0,00 & - & 1 & 1,39 & 1 & 1,39 & - \\
\hline \multicolumn{12}{|c|}{ Triquíase/ Ectrópio } \\
\hline Sim & & 0 & 0 & 0 & 0,00 & - & 1 & 1,45 & 1 & 1,39 & 0,9999 \\
\hline Não & & 18 & 25,00 & 18 & 25,00 & 0,9999 & 2 & 2,90 & 2 & 2,78 & \\
\hline Não/ Não & & 54 & 75,00 & 54 & 75,00 & & 69 & 95,83 & 69 & 95,83 & \\
\hline \multicolumn{12}{|c|}{ Diminuição da sensibilidade } \\
\hline \multicolumn{12}{|l|}{ Córnea } \\
\hline Sim & & 1 & 1,39 & 3 & 4,17 & $<0,001$ & 0 & 0 & 0 & 0 & - \\
\hline Não & 71 & & 98,61 & 69 & 95,83 & & 72 & 100 & 72 & 100 & \\
\hline \multicolumn{12}{|c|}{ Opacidade córnea } \\
\hline Sim & 1 & & 1,39 & 1 & 1,39 & 0,9999 & 0 & 0 & 0 & 0 & - \\
\hline Não & 71 & & 98,61 & 71 & 98,61 & & 72 & 100 & 72 & 100 & \\
\hline \multicolumn{12}{|l|}{ Catarata } \\
\hline Sim & 1 & & 1,39 & 2 & 2,78 & $<0,001$ & 1 & 1,39 & 2 & 2,78 & $<0,001$ \\
\hline Não & 70 & & 97,22 & 69 & 95,83 & & 69 & 95,83 & 69 & 95,83 & \\
\hline Não Avaliado & 1 & & 1,39 & 1 & 1,39 & & 2 & 2,78 & 2 & 2,78 & \\
\hline
\end{tabular}

Fonte: Dados da pesquisa. Elaborada pelos autores.

O perfil dos casos de hanseníase referente a avaliação dos membros superiores no diagnóstico foi: sem queixas $(65,28 \%)$, ulnar normal $(87,56 \%)$, mediano lado direito $(80,56 \%)$ e esquerdo (76,39\%), radial direito $(90,28 \%)$ e esquerdo $(91,67 \%)$. Quanto avaliação de força ao abrir o dedo mínimo forte lado direito $(73,61 \%)$ e esquerdo $(77,78)$, elevar o polegar forte lado direito $(81,94 \%)$ e esquerdo (80,56\%), elevar o punho forte lado direito $(98,83 \%)$ e esquerdo $(93,06 \%)$ (Tabela 4$)$.

O perfil dos casos de hanseníase referente a avaliação dos membros superiores na alta foi: sem queixas lado direito $(83,33 \%)$ e lado esquerdo $(81,94 \%)$, ulnar normal lado direito $(88,89 \%)$ e lado esquerdo $(88,89 \%)$, mediano lado direito $(79,17 \%)$ e esquerdo $(80,56 \%)$, radial em ambos os lados $(95,83 \%)$. Quanto avaliação de força ao abrir o dedo mínimo forte lado direito $(83,33 \%)$ e esquerdo $(77,78)$, elevar o polegar forte lado direito $(84,72 \%)$ e esquerdo $(90,28 \%)$, elevar o punho forte lado direito $(95,83 \%)$ e esquerdo $(94,44 \%)$ (Tabela 4$)$.

Houve significância estatística com os seguintes resultados: sem queixas na alta, nervo ulnar no diagnóstico, mediano no diagnóstico e na alta, avaliação de força ao abrir o dedo mínimo no diagnóstico e na alta e elevar o polegar na alta (Tabela 4). 
Tabela 4. Comparação da avaliação dos membros superiores durante o diagnóstico e alta dos casos de hanseníase com Grau de Incapacidade Física (GIF) dos anos 2017 e 2018. Rondonópolis (MT), 2020.

\begin{tabular}{|c|c|c|c|c|c|c|c|c|c|c|}
\hline \multirow[t]{3}{*}{ Membros superiores } & \multicolumn{4}{|c|}{ Diagnóstico } & \multirow{3}{*}{ Valor $\mathbf{p}$} & \multirow{2}{*}{\multicolumn{2}{|c|}{ Direito }} & \multirow{2}{*}{\multicolumn{2}{|c|}{$\begin{array}{l}\text { Alta } \\
\text { Esquerdo }\end{array}$}} & \multirow{3}{*}{ Valor $\mathbf{p}$} \\
\hline & \multicolumn{2}{|c|}{ Direito } & \multicolumn{2}{|c|}{ Esquerdo } & & & & & & \\
\hline & $\mathbf{N}$ & $\%$ & $\mathbf{N}$ & $\%$ & & $\mathbf{N}$ & $\%$ & $\mathbf{N}$ & $\%$ & \\
\hline \multicolumn{11}{|l|}{ Queixa principal } \\
\hline Sem queixas & 47 & 65,28 & 47 & 65,28 & 0,9765 & 60 & 83,33 & 59 & 81,94 & $<0,001$ \\
\hline Dor & 3 & 4,17 & 3 & 4,17 & & 4 & 5,56 & 4 & 5,56 & \\
\hline Alteração de força & 4 & 5,56 & 4 & 5,56 & & 2 & 2,78 & 1 & 1,39 & \\
\hline Dormência & 15 & 20,83 & 16 & 22,22 & & 2 & 2,78 & 5 & 6,94 & \\
\hline Inchaço & 2 & 2,78 & 2 & 2,78 & & 1 & 1,39 & 1 & 1,39 & \\
\hline Em branco/ignorado & 1 & 1,39 & 0 & 0 & - & 3 & 4,17 & 2 & 2,78 & - \\
\hline \multicolumn{11}{|l|}{ Ulnar } \\
\hline Normal & 63 & 87,5 & 63 & 87,50 & $<0,001$ & 64 & 88,89 & 64 & 88,89 & 0,9865 \\
\hline Dor & 9 & 12,5 & 7 & 9,72 & & 4 & 5,56 & 4 & 5,56 & \\
\hline Espessado & 9 & 12,5 & 7 & 9,72 & & 3 & 4,17 & 4 & 5,56 & \\
\hline Dor e choque & 0 & 0 & 2 & 2,78 & - & 0 & 0 & 0 & 0 & - \\
\hline Dor e espessado & 0 & 0 & 0 & 0 & - & 1 & 1,39 & 0 & 0 & - \\
\hline \multicolumn{11}{|l|}{ Mediano } \\
\hline Normal & 58 & 80,56 & 55 & 76,39 & $<0,001$ & 57 & 79,17 & 58 & 80,56 & $<0,001$ \\
\hline Dor & 9 & 12,50 & 14 & 19,44 & & 9 & 12,50 & 9 & 12,50 & \\
\hline Espessado & 0 & 0 & 0 & 0 & - & 2 & 2,78 & 0 & 0 & \\
\hline Dor e espessado & 0 & 0 & 0 & 0 & - & 0 & 0 & 1 & 1,39 & \\
\hline Dor e choque & 5 & 6,94 & 3 & 4,17 & & 1 & 1,39 & 1 & 1,39 & \\
\hline Não avaliado & 0 & 0 & 0 & 0 & & 3 & 4,17 & 3 & 4,17 & \\
\hline \multicolumn{11}{|l|}{ Radial } \\
\hline Normal & 65 & 90,28 & 66 & 91,67 & 0,0745 & 69 & 95,83 & 69 & 95,83 & 0,4532 \\
\hline Dor & 7 & 9,72 & 6 & 8,33 & & 2 & 2,78 & 2 & 2,78 & \\
\hline Dor e choque & 0 & 0 & 0 & 0 & & 1 & 1,39 & 1 & 1,39 & \\
\hline \multicolumn{11}{|l|}{ Avaliação de força } \\
\hline \multicolumn{11}{|l|}{ Abrir dedo mínimo } \\
\hline Forte & 53 & 73,61 & 56 & 77,78 & $<0,001$ & 60 & 83,33 & 56 & 77,78 & $<0,001$ \\
\hline Diminuído & 19 & 26,39 & 15 & 20,83 & & 11 & 15,28 & 15 & 20,83 & \\
\hline Não avaliado & 0 & 0 & 1 & 1,39 & - & 1 & 1,39 & 1 & 1,39 & - \\
\hline \multicolumn{11}{|l|}{ Elevar polegar } \\
\hline Forte & 59 & 81,94 & 58 & 80,56 & 0,0879 & 61 & 84,72 & 65 & 90,28 & $<0,001$ \\
\hline Diminuído & 13 & 18,06 & 13 & 18,06 & & 10 & 13,89 & 5 & 6,94 & \\
\hline Não avaliado & 0 & 0 & 1 & 1,39 & & 1 & 1,39 & 2 & 2,78 & \\
\hline \multicolumn{11}{|l|}{ Elevar punho } \\
\hline Forte & 69 & 95,83 & 67 & 93,06 & 0,0976 & 69 & 95,83 & 68 & 94,44 & 0,0774 \\
\hline Diminuído & 1 & 1,39 & 1 & 1,39 & & 2 & 2,78 & 2 & 2,78 & \\
\hline Não avaliado & 2 & 2,78 & 2 & 2,78 & - & 1 & 1,39 & 2 & 2,78 & - \\
\hline
\end{tabular}

Fonte: Dados da pesquisa. Elaborada pelos autores. 
O perfil dos casos de hanseníase referente a avaliação dos membros inferiores no diagnóstico foi: sem queixas (54,17\%), fibular normal lado direito (76,39\%) lado esquerdo (70,83\%), tibial posterior lado direito $(76,39 \%)$ e lado esquerdo $(77,78 \%)$. Com relação a avaliação de força ao elevar o halux foi forte $(87,50 \%)$, elevar o pé foi forte sendo lado direito $(95,83 \%)$ e lado esquerdo $(91,67)$ (Tabela 5).

O perfil dos casos de hanseníase referente a avaliação dos membros inferiores na alta foi: sem queixas lado direito $(69,44 \%)$ e lado esquerdo (70,83\%), fibular normal lado direito $(90,28 \%)$ lado esquerdo $(87,50 \%)$, tibial posterior ambos os lados $(86,11 \%)$. Com relação a avaliação da força ao elevar o halux, foi forte $(90,28 \%)$, elevar o pé permaneceu forte sendo lado direito $(95,83 \%)$ e lado esquerdo $(90,28)$ (Tabela 5). A variável com significância estatística foi a avaliação fibular na alta. 
Tabela 5. Comparação da avaliação dos membros inferiores durante o diagnóstico e alta dos casos de hanseníase com Grau de Incapacidade Física (GIF) dos anos 2017 e 2018. Rondonópolis (MT), 2020.

\begin{tabular}{|c|c|c|c|c|c|c|c|c|c|c|}
\hline \multirow[t]{3}{*}{ Membros Inferiores } & \multicolumn{4}{|c|}{ Diagnóstico } & \multicolumn{6}{|c|}{ Alta } \\
\hline & \multicolumn{2}{|l|}{ Direito } & \multicolumn{2}{|c|}{ Esquerdo } & \multicolumn{3}{|c|}{ Valor pDireito } & \multicolumn{2}{|c|}{ Esquerdo } & \multirow[t]{2}{*}{ Valor $\mathbf{p}$} \\
\hline & $\mathbf{N}$ & $\%$ & $\mathbf{N}$ & $\%$ & & $\mathbf{N}$ & $\%$ & $\mathbf{N}$ & $\%$ & \\
\hline \multicolumn{11}{|l|}{ Queixa principal } \\
\hline Sem queixas & 39 & 54,17 & 39 & 54,17 & 0,0977 & 50 & 69,44 & 51 & 70,83 & 0,0993 \\
\hline Dor & 11 & 15,28 & 10 & 13,89 & & 2 & 2,78 & 4 & 5,56 & \\
\hline Alteração de força & 6 & 8,33 & 7 & 9,72 & & 3 & 4,17 & 3 & 4,17 & \\
\hline Formigamento & 3 & 4,17 & 2 & 2,78 & & 0 & 0 & 0 & 0 & \\
\hline Dormência & 11 & 15,28 & 12 & 16,67 & & 13 & 18,06 & 9 & 12,50 & \\
\hline Inchaço & 1 & 1,39 & 0 & 0 & & 1 & 1,39 & 1 & 1,39 & \\
\hline Câimbra & 1 & 1,39 & 1 & 1,39 & & 1 & 1,39 & 2 & 2,78 & \\
\hline Em branco/ignorado & 0 & 0 & 1 & 1,39 & - & 2 & 2,78 & 2 & 2,78 & - \\
\hline \multicolumn{11}{|l|}{ Fibular } \\
\hline Normal & 55 & 76,39 & 51 & 70,83 & 0,0793 & 65 & 90,28 & 63 & 87,50 & 0,0456 \\
\hline Dor & 10 & 13,89 & 13 & 18,06 & & 5 & 6,94 & 7 & 9,72 & \\
\hline Espessado & 3 & 4,17 & 5 & 6,94 & & 2 & 2,78 & 2 & 2,78 & \\
\hline Dor e espessado & 3 & 4,17 & 2 & 2,78 & & 0 & 0 & 0 & 0 & \\
\hline Dor e choque & 1 & 1,39 & 1 & 1,39 & & 0 & 0 & 0 & 0 & \\
\hline \multicolumn{11}{|l|}{ Tibial Posterior } \\
\hline Normal & 55 & 76,39 & 56 & 77,78 & 0,0654 & 62 & 86,11 & 62 & 86,11 & 0,9150 \\
\hline Dor & 12 & 16,67 & 13 & 18,06 & & 8 & 11,11 & 8 & 11,11 & \\
\hline Espessado & 2 & 2,78 & 1 & 1,39 & & 1 & 1,39 & 0 & 0 & \\
\hline Dor e espessado & 2 & 2,78 & 1 & 1,39 & & 1 & 1,39 & 2 & 2,78 & \\
\hline Dor e choque & 1 & 1,39 & 1 & 1,39 & & 0 & 0 & 0 & 0 & \\
\hline \multicolumn{11}{|c|}{ AVALIAÇÃO DE FORÇA } \\
\hline \multicolumn{11}{|l|}{ Elevar o halux } \\
\hline Forte & 63 & 87,50 & 63 & 87,50 & 0,9999 & 65 & 90,28 & 65 & 90,28 & 0,9998 \\
\hline Diminuído & 8 & 11,11 & 8 & 11,11 & & 5 & 6,94 & 6 & 8,33 & \\
\hline Paralisado & 0 & 0 & 0 & 0 & & 1 & 1,39 & 0 & 0 & \\
\hline Não avaliado & 1 & 1,39 & 1 & 1,39 & - & 1 & 1,39 & 1 & 1,39 & - \\
\hline \multicolumn{11}{|l|}{ Elevar o pé } \\
\hline Forte & 69 & 95,83 & 66 & 91,67 & 0,0876 & 69 & 95,83 & 65 & 90,28 & 0,0856 \\
\hline Diminuído & 3 & 4,17 & 4 & 5,56 & & 3 & 4,17 & 6 & 8,33 & \\
\hline Não avaliado & 0 & 0 & 2 & 2,78 & - & 0 & 0 & 1 & 1,39 & - \\
\hline
\end{tabular}

Fonte:|Dados da pesquisa. Elaborada pelos autores.

\section{DISCUSSÕES}

Conforme os resultados da pesquisa desenvolvida, mais da metade dos usuários com hanseníase no diagnóstico $(73,61 \%)$ e na alta $(84,72 \%)$ não tinham alteração de capacidade física. Em estudo realizado em Botucatu (SP), ao avaliar a alteração do GIF, ressaltou-se que grande parte da população estudada também não apresentava alteração (65\%) (DUARTE et al., 2007). Na Bahia, não foram encontradas incapacidades em $81,5 \%$ dos pacientes na avaliação inicial (PINTO et al., 2011). 
Estudos demonstram que, aproximadamente, $20 \%$ do total dos usuários que são casos novos de hanseníase desenvolvem alguma alteração física e cerca de $15 \%$ ao longo do tratamento, provavelmente, terão algum GIF, mesmo com o cuidado com as neurites, estados reacionais e fazendo uso da PQT (DUERKSEN, 2000; DUERKSEN, 2003). Apesar de ser considerado um município endêmico para hanseníase, no que diz respeito ao GIF, Rondonópolis possui indicadores melhores do que está preconizado pelo Ministério da Saúde, que determina que 23\% dos acometidos pela doença, recebem alta com presença de alteração da capacidade física (BRASIL, 1999).

Neste estudo, verificou-se que houve aumento dos casos com GIF0 e diminuição dos casos com GIF1 e GIF2 quando comparados diagnóstico e alta. Além disso, houve um valor acentuado do GIF 1 e GIF 2 no diagnóstico (25\%) e na cura (15,28\%) dos casos de hanseníase e houve na alta uma redução dos casos com GIF 1 (de 19,44\% no diagnóstico para 9,72\%). Esta diminuição nos GIF do diagnóstico até a alta foi descrita em Tangará da Serra (MT), entre 2007 a 2016, GIF 1 (8,34\%) e GIF 2 (1,68\%) (MARQUES et al., 2017) e em Duque de Caxias (RJ) com redução da proporção de casos com GIF de 13,6\% para 8,6\% (DUARTE-CUNHA et al., 2012). Por outro lado, em Várzea Grande (MT) houve piora do quadro quando comparado o GIF no momento da alta com o diagnóstico, onde encontrou-se indivíduos que finalizaram o tratamento sem incapacidade evoluíram para GIF 1 $(26,2 \%)$ e os com GIF 1 agravaram para GIF 2 (5,8\%) (RAMOS; SOUTO, 2010).

Além disso, houve significância estatística do número de casos com GIF tanto no diagnóstico como na alta dos usuários em Rondonópolis (MT). Fato que contradiz com um estudo realizado na Paraíba no período de 2009 a 2014, em que ao realizar esta comparação nos casos com GIF, não foi encontrada diferença estatística significativa $(\mathrm{p}=0,148)$ (SANTANA et al., 2018).

Os casos de hanseníase com GIF neste estudo prevaleceram sexo masculino, adultos, profissionais liberais, baixa escolaridade, residentes na zona urbana e pardos. Alguns estudos evidenciaram a prevalência de algumas destas características com GIF como na Paraíba, onde prevaleceram homens (59\%), faixa etária de 31 a 45 anos (27,8\%) e ensino fundamental (53,6\%) (SANTANA et al., 2018); em Campo Grande (MS) homens (59,4\%), adultos $(62,3 \%)$ e ensino fundamental (84\%) (BERNARDES et al., 2009) e em São Luís (MA) adultos (74,9\%), autônomo $(18,7 \%)$, baixa escolaridade $(36,8 \%)$ e pardos (58,6\%) (ARAÚJO et al., 2014). O usuário com perfil socioeconômico mais vulnerável, reflete na presença de incapacidade física interferindo negativamente na qualidade de vida (DUARTE et al., 2007).

Somente a variável sexo não apresentou correlação estatística com os casos de GIF neste estudo, no entanto, em Belo Horizonte (MG), de 1993 a 2003, outras variáveis sociodemográficas como ocupação, sexo, idade e região de procedência, não se associaram à evolução do grau de incapacidade (GONÇALVES et al., 2009), já em Campo Grande (MS) observou-se associação estatisticamente significativa entre sexo e o GIF (BERNARDES et al., 2009).

No que diz respeito ao perfil clínico epidemiológico dos casos de hanseníase com GIF neste estudo prevaleceu casos novos, modo de detecção por encaminhamento, baciloscopia não realizada, classificação multibacilar e forma clínica dimorfa. Houve predomínio dessas variáveis com diferença estatística para os GIF dos casos. 
Perfil semelhante encontrado nos estudos em Campo Grande (MS) com encaminhamento dos casos $(50 \%)$ e multibacilar $(55,7 \%)$ com associação estatística $(p<0,001)$ (CORRÊA; IVO \& HONER, 2006). Em Araguaína (TO) houve associação entre incapacidades físicas com classificação multibacilar ( $<$ 0,001) (MONTEIRO et al., 2013). Em São José do Rio Preto (SP) o GIF foi mais frequente nas formas clínicas dimorfa e virchoviana em relação a outras (NARDI et al., 2012). Outro estudo em Campo Grande (MS) traz a prevalência da forma multibacilar (51,6\%) e associação estatisticamente significativa entre a forma clínica e o GIF (BERNARDES et al., 2009).

Neste estudo foi analisada a avaliação da face no diagnóstico e alta com ressalvas para a região do nariz sem queixas, ausência de ressecamento e ferida e região dos olhos sem queixas, fechamento dos olhos, triquíase e ectrópio sem alteração, diminuição da sensibilidade e opacidade córnea sem alteração e catarata sem alterações. Somente a diminuição da sensibilidade córnea durante o diagnóstico e catarata durante o diagnóstico e a alta tiveram significância estatística.

Esta avaliação realizada na Paraíba apontou decréscimo de comprometimento ao comparar o diagnóstico e a alta por cura com diferença estatisticamente significativa apenas para o nariz, ressecamento e ferida (SANTANA et al., 2018). Em São Luís (MA) no diagnóstico 51,6\% possuíam complicações nos olhos e, após o tratamento, houve diminuição (45,8\%) e no nariz, 7,1\% apresentaram complicações ao diagnóstico e ao final do tratamento 11\% (ARAUJO et al., 2014). Em Presidente Prudente (SP), somente 5\% dos casos tiveram alterações nos olhos, não sendo identificada associação estatística (FARIA et al., 2015).

Além disso, neste estudo foram avaliados os membros superiores no diagnóstico e alta com prevalência de sem queixas, normalidade de nervos ulnar, mediano e radial, força ao abrir o dedo mínimo, elevar o polegar forte e elevar o punho forte. Houve significância estatística nas variáveis sem queixas na alta, nervo ulnar no diagnóstico, mediano no diagnóstico e na alta, avaliação de força ao abrir o dedo mínimo no diagnóstico e na alta e elevar o polegar na alta.

No que diz respeito às alterações dos membros superiores, em São Luís (MA), apresentaram complicações no início do tratamento nos nervos radial (18,7\%), mediano (9,7\%) e ulnar (11\%); mais um trabalho que compara início e alta do tratamento, houve significância estatística apenas para aquelas observadas no nervo radial (ARAUJO et al., 2014). Em Uberlândia (MG), prevalece a alteração no nervo ulnar, sendo o mais acometido tanto no diagnóstico $(22,3 \%)$ como na alta $(23,9 \%)$ (KIL et al., 2012). Outro estudo no Rio de Janeiro (RJ), no qual 67\% dos usuários apresentaram acometimento nervoso, constatou-se correlação estatisticamente significativa entre a presença de nervos periféricos acometidos e incapacidades físicas no momento do diagnóstico (PIMENTEL et al., 2003).

Em relação a avaliação dos membros inferiores no diagnóstico e alta predominaram sem queixas, normalidade de fibular e tibial posterior, avaliação de força ao elevar o halux e o pé forte. Somente a variável com significância estatística foi a avaliação fibular na alta. Em Brasília (DF) prevaleceram alterações nos nervos fibular direito (18,5\%) e tibial posterior bilateralmente $(22,2 \%)$ durante o tratamento (CARVALHO; ALVAREZ, 2000); em Uberlândia (MG) destacaram as alterações no nervo fibular tanto no diagnóstico (20,7\%) como na alta (21,3\%) (KIL et al., 2012); na Paraíba foi 
observado diminuição do comprometimento de todos os nervos do diagnóstico para alta, apresentando diferença estatisticamente significativa, exceto para o nervo mediano $(\mathrm{p}=0,056)$ (SANTANA et al., 2018).

\section{CONCLUSÃO}

Neste estudo foi possível evidenciar que a maioria dos pacientes com hanseníase foram diagnosticados precocemente, visto que mais da metade da amostra não apresentou alteração de capacidade física tanto no diagnóstico como na alta. Foram observadas mudanças significantes na presença de incapacidade física e das deficiências.

Embora a presença de dados positivos mencionados com relação ao GIF, é importante ressaltar que uma parcela dos usuários recebeu alta com algum grau de incapacidade um ou dois, sendo fundamental o acompanhamento destes para que não venham piorar seu estado de saúde. Outro ponto de atenção é quanto à necessidade de desenvolvimento de um protocolo de seguimento dos pacientes após o tratamento.

Neste contexto é fundamental a avaliação das políticas públicas e da rede de serviços no município em estudo que sejam direcionadas para o tratamento da hanseníase, sendo necessário o desenvolvimento de estratégias voltadas para o diagnóstico precoce, prevenção de incapacidade física e deficiências, tendo em vista a possibilidade de piora do grau de incapacidade durante o tratamento.

Portanto medidas preventivas, como educação em saúde, diagnóstico precoce e busca ativa de novos casos, voltadas às incapacidades são essenciais e devem fazer parte da rotina do tratamento, considerando as sequelas físicas, psicológicas e sociais, que a hanseníase pode desencadear influenciando na qualidade de vida do indivíduo. Além disso, a abordagem multiprofissional é fundamental nesse processo de reinserção do usuário na sociedade, contribuindo para um tratamento eficaz e com menos prejuízos a sua saúde.

Este estudo apresentou como limitação a coleta através de dados documental com a ausência do preenchimento correto da ficha de avaliação neurológica e de dados sobre os nervos afetados e número de lesões cutâneas que poderiam acrescentar informações. Algumas fichas não foram preenchidas, devido ausência de um profissional capacitado para realização das avaliações dermatoneurológicas e devido a isso não foram inseridas na população do estudo.

Por fim os dados do presente estudo trazem embasamento para melhoria do serviço de saúde voltado a hanseníase, corroborando com o fomento de novas pesquisas, bem como contribuindo para a melhora do quadro endêmico do município.

\section{DECLARAÇÃO DE INTERESSES}

Nós, autores deste artigo, declaramos que não possuímos conflitos de interesses de ordem financeira, comercial, político, acadêmico e pessoal. 


\section{REFERÊNCIAS}

ARAUJO, A.E.R.A.; AQUINO, D.M.C.; GOULART, I.M.B.; PEREIRA, S.R.F.; FIGUEIREDO, I.A.; SERRA, H.O; ; et al. Neural complications and physical disabilities in leprosy in a capital of northeastern Brazil with high endemicity. Rev Bras Epidemiol, v. 17, n. 4, p. 899-910, 2014.

BERNARDES, C.A.; SANTOS, A.F.; PADOVANI, C.T.J.; SANTOS, L.F.; HANS FILHO, G. Physical disability in leprosy patients in Campo Grande - Mato Grosso do Sul. Hansen Int, v. 34 n. 1, p. 17-25, 2009.

BRASIL. Ministério da Saúde. Secretaria de Políticas de Saúde. Departamento de estão de Políticas Estratégicas. Relatório de atividades da área técnica de dermatologia sanitária. Brasília: Ministério da Saúde, 1999.

BRASIL. Ministério da Saúde. Área Técnica de Dermatologia Sanitária. Hanseníase atividades de controle e manual de procedimentos. Brasília (DF): Ministério da Saúde, 2004.

BRASIL. Ministério da Saúde. Secretaria de Vigilância em Saúde. Departamento de Vigilância Epidemiológica. Manual de Prevenção de Incapacidades. Série A. Normas e Manuais Técnicos. Cadernos de Prevenção e Reabilitação em hanseníase, n.1. Brasília (DF): Ministério da Saúde, 2008.

BRASIL. Conselho Nacional de Saúde. Resolução $\mathbf{n}^{\circ}$ 466, de 12 de dezembro de 2012. Aprova normas regulamentadoras de pesquisas envolvendo seres humanos. Brasília: Diário Oficial da União, 2012.

BRASIL. Ministério da Saúde. Secretaria de Vigilância em Saúde. Departamento de Doenças Transmissíveis. Diretrizes para vigilância, atenção e eliminação da Hanseníase como problema de saúde pública: manual técnico-operacional. Brasília (DF): Ministério da Saúde, 2016.

BRASIL. Ministério da Saúde. Secretaria de Vigilância em Saúde. Departamento de Vigilância das Doenças Transmissíveis. Guia Prático Sobre a Hanseníase. Brasília (DF): Ministério da Saúde, 2017.

BRASIL. Ministério da Saúde. Estratégia Nacional para o Enfrentamento da Hanseníase 2019 2022. Brasília (DF): Ministério da Saúde, 2019.

BRASIL. Ministério da Saúde. Secretaria de Vigilância em Saúde. Boletim Epidemiológico Hanseníase. n. especial, 2020, Editora MS/CGDI, Brasília, 2020.

CARVALHO, G.A.; ALVAREZ, R.R.A. Avaliação de Incapacidades de Incapacidades Físicas Neuro-Músculo-Esquelética em Pacientes com Hanseníase. Hansen Int., v. 25, n. 1, p. 39-48, 2000.

CORRÊA, C.M.J.; IVO, M. L.; HONER M. R. Incapacidades em sujeitos com hanseníase em um centro de referência do centro-oeste brasileiro entre 2000-2002. Hansen Int., v. 31, n. 2, p. 71-89, 2006.

DUARTE, M.T.C.; AYRES, J.A.; SIMONETTI, J.P. Socioeconomic and demographic profile of leprosy carriers attended in nursing consultations. Revista Latino-Americana de Enfermagem, v. 
15, n. especial, p. 774-779, 2007.

DUARTE-CUNHA, M.; SOUZA-SANTOS, R.; MATOS, H.J.; OLIVEIRA, M.L.W. Aspectos epidemiológicos da hanseníase: uma abordagem espacial. Cad. Saúde Pública, v.28, n.6, p.11431155, 2012.

DUERKSEN, F. Reabilitação. In: Opromolla DVA, editor. Noções de Hansenologia. Bauru: Centro de Estudos Dr. Reynaldo Quagliato, Instituto Lauro de Souza Lima, p.113-115, 2000.

DUERKSEN, F. Reabilitação. In: Opromolla DVA, Baccarelli R, editores. Prevenção de incapacidades e reabilitação em hanseníase. Bauru, Instituto Lauro Souza Lima, p. 3- 4, 2003.

FARIA, C.R.S.; FREGONESI, C.E.P.T.; CORAZA, D.A.G.; ANDRADE, D.M.; MANTOVANI, N.A.D.T.; SILVA, J.R.; et al. Grau de incapacidade física de portadores de hanseníase: estudo de coorte retrospectivo. Arquivos de Ciências da Saúde, v.22, n.4, p.58-62, 2015.

GONÇALVES, S.D.; SAMPAIO, R.F.; ANTUNES, C.M.F. Predictive factors of disability in patients with leprosy. Rev. Saúde Pública, v. 43, n. 2, p.1-8, 2009.

KIL, A.K.A.; SILVESTRE, C.M.; KAMINICE, L.M.; QUINTINO, L.B.; LIMA, L.B.; PARANHOS, M.B.; et al. Deficiências e incapacidades por Hanseníase: Avaliação clínica e epidemiológica dos pacientes atendidos em um Centro de Referência Nacional do Brasil. Hansen Int, v.37, n.1, p.25-33, 2012.

MARQUES, M.S.; CABRAL, J.F.; TERÇAS, A.C.P.; SANTANA, D.P.; SILVA, J.H. Clinical and epidemiological leprosy profile in the ciy of Tangará da Serra, Mato Grosso. Revista Norte Mineira de Enfermagem, v. 6, n. 2, p. 34-47, 2017.

MATO GROSSO. Secretaria de Estado de Saúde. Plano estratégico para enfrentamento da hanseníase, 2018.

MONTEIRO, L.D.; ALENCAR, C.H.M.; BARBOSA, J.C.; BRAGA, K.P.; CASTRO, M.D.; HEUKELBACH, J. Incapacidades físicas em pessoas acometidas pela hanseníase no período pós-alta da poliquimioterapia em um município no Norte do Brasil. Cad Saúde Pública, v. 29, p. 909-920, 2013.

NARDI, S.M.T.; PASCHOAL, V.D’A; CHIARAVALlOTI-NETO, F.; ZANETTA, D.M.T. Deficiências após a alta medicamentosa da hanseníase: prevalência e distribuição espacial. Rev. Saúde Pública, v.46, n.6, p. 969-77, 2012.

OMS. Organização Mundial da Saúde. Global leprosy update, 2018: moving towards a leprosy free world. Weekly Epidemiological Record. Genebra, n. 94, p. 389-412, 2019.

PIMENTEL, M.I.F.; NERY, J.A.C.; BORGES, E.; GONÇALVES, R.R.; SARNO, E.M. Exame neurológico inicial na hanseníase multibacilar: correlação entre a presença de nervos afetados com incapacidades presentes no diagnóstico e com a ocorrência de neurites francas. An bras Dermatol. v.78, n. 5, p. 561-7, 2003. 
PINTO, R.A.; MAIA, H.F.; SILVA, M.A.F.; MARBACK, M. Perfil clínico e epidemiológico dos pacientes notificados com hanseníase em um hospital especializado em Salvador, Bahia. Rev B. S. Publica, v. 34, n. 4, p.906-918, 2011.

$\mathrm{R}$ Core Team 2020. A language and environment for statistical computing. R Foundation for Statistical Computing, Vienna, Austria. Disponível em: http:/www.R- project.org/.

RAMOS, J.M.H.; SOUTO, F.J.D. Incapacidade pós-tratamento em pacientes hansenianos em Várzea Grande, estado de Mato Grosso. Revista da Sociedade Brasileira de Medicina Tropical, v. 43, n. 3, p. 293-297, 2010.

RIBEIRO, G.C.; LANA, F.C.F. Incapacidades físicas em hanseníase: caracterização, fatores relacionados e evolução. Revista Cogitare Enfermagem, v. 20, n.3, p. 486 -503, 2015.

SANTANA E.M.F.; BRITO, K.K.G.; NOGUEIRA, J.A.N.; LEADEBAL, O.D.C.P.; COSTA, M.M.L.; et al. Deficiências e incapacidades na hanseníase: do diagnóstico à alta por cura. Rev. Eletr. Enf, v. 20, n. 15, p. 1-11, 2018.

SILVA SOBRINHO, R.A.; MATHIAS, T.A.F.; GOMES, E.A.; LINCOLN, P.B. Avaliação do grau de incapacidade em hanseníase: uma estratégia para sensibilização e capacitação da equipe de enfermagem. Rev. Latino-Am. Enferm., v.15, n.6, p. 1125-30, 2007. 


\section{Índice Remissivo}

A

Ansiedade 70, 71, 72, 74, 76, 77, 80, 81

Assistência materno-fetal 99

Atenção primária a saúde (aps) 99

Atendimento à mulher grávida 24, 34

C

Cenário pandêmico 70

Condições de trabalho 89, 91, 92, 93

Covid-19 entre mulheres 58

Covid-19 (sars-cov-2) 70, 71

Crise global 58, 67

Cuidados de enfermagem 99, 103

D

Dcv relacionadas ao trabalho 89,91

Degeneração neural 40

De pacientes pos-cirúrgicos 106

Departamento de condições crônicas e infecções sexualmente transmissíveis (dcci) 99

Depressão 70, 71, 72, 76, 77, 80, 81

Desenvolvimento de saúde feminina 11

Diretrizes do ministério da saúde 24, 34

Doenças cardiovasculares 89, 96

Doenças cardiovasculares em trabalhadores 89,91

E

Estresse $70,71,72,73,74,75,78,79,80,92,93,94,95$

G

Gestão em saúde 58

Gravidez 14, 24

$\mathrm{H}$

Hanseníase 6, 39, 40, 41, 42, 43, 44, 45, 46, 47, 48, 49, 50, 51, 53, 54, 55, 56

Hemácias 106, 107, 108, 112, 114

Hemotransfusão 6, 106, 108, 109, 111, 113, 114

I

Impactos psicossociais da pandemia de covid-19 70

Impactos socioeconômicos 70, 79, 80

Incapacidades físicas 39, 41, 52

Indicadores e fortalecimento das políticas 11

Índice de desenvolvimento humano (idh) 58 
Investimento governamental 11,22

Isolamento social $70,71,78$

$\mathrm{L}$

Lesões genitais 99

$\mathrm{M}$

Mortalidade materna 6, 7, 11, 12, 20, 21, 23, 24, 26, 27, 28, 31, 32, 33, 34, 35, 36, 37

$\mathrm{O}$

O adoecimento dos trabalhadores 89

Óbitos maternos 11, 13, 21, 24, 26, 27, 28, 29, 30, 32, 33, 34, 35

Óbitos relacionados à covid-19 58

$\mathrm{P}$

Pandemia da covid-19 58, 74

Parto natural 99

Perfil das mulheres 11

Perfil de saúde 106

Políticas públicas vigentes 11,21

Pré-natal 99, 103, 104

$\mathrm{R}$

Rede materno infantil 24, 32, 34, 35

S

Sangue 74, 106, 110, 112, 114, 115, 117

Saúde da mulher 12, 18, 20, 24, 32, 95

Saúde do trabalhador 89, 96

Saúde mental dos indivíduos 70

Sequelas permanentes $39,40,41$

Sífilis congênita (sc) 99

$\mathrm{T}$

Trabalho 89, 93, 96

Transfusões de sangue 106

Treponema pallidum 99, 100

$\mathrm{U}$

Uso de substâncias 70, 72, 76, 79, 80

$\mathrm{V}$

Variação dos óbitos 11, 21

Vítimas de morte materna 24, 26, 33 


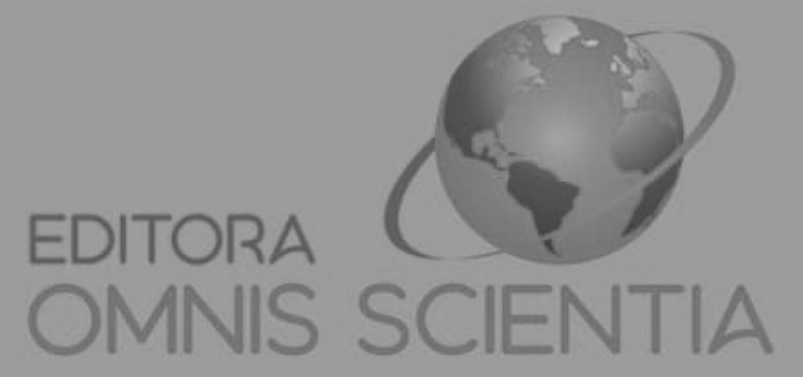

editoraomnisscientia@gmail.com M https://editoraomnisscientia.com.br/ (-) @editora_omnis_scientia (0) https://www.facebook.com/omnis.scientia.9 f +55 (87) 9656-3565 @ 
editoraomnisscientia@gmail.com

https://editoraomnisscientia.com.br/

@editora_omnis_scientia

+55 (87) 9656-3565 